\title{
Pleomorphic Adenoma of Palate - A Case Report
}

\section{S. Sakthivel 1, ", Caroline Rajprabhu ${ }^{2}$, V. Rajprabhu ${ }^{3}$, C. Gokula Krishan ${ }^{4}$}

${ }^{1}$ MDS OMFS, Velss dental care, Chennai, India.

${ }^{2}$ MDS periodontics, Mylai dental and implant centre, Mylapore Chennai, India.

${ }^{3}$ MDS OMFS, Mylai dental and implant centre, Mylapore, Chennai, India.

${ }^{4}$ MDS OMFS, C.R. Dental and implant centre, Valasaravakkam, Chennai, India.

*Corresponding Author: V. S. Sakthivel, MDS OMFS, Velss Dental care, Chennai, India.

Received date: 17 April 2021; Accepted date: 10 May 2021; Published date: 12 May 2021

Citation: Sakthivel VS, Rajprabhu C, Rajprabhu V, Krishan CG. Pleomorphic Adenoma of Palate - A Case Report. J Med Case Rep Case Series 2(3): https://doi.org/10.38207/jmcrcs20210047

Copyright: (C) 2021 V. S. Sakthivel. This is an open-access article distributed under the terms of the Creative Commons Attribution License, which permits unrestricted use, distribution, and reproduction in any medium, provided the original author and source are credited.

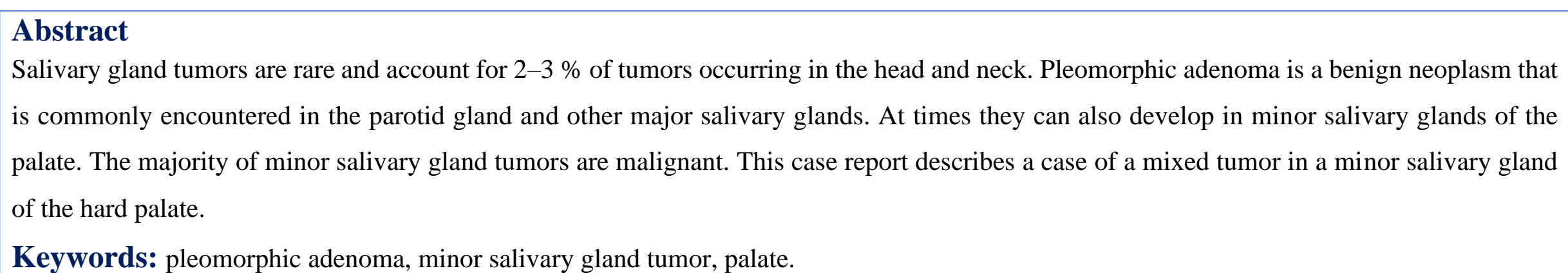

\section{Introduction}

Pleomorphic adenoma (PA), which is the most common tumor of salivary glands, is a benign neoplasm consisting of cells exhibiting the ability to differentiate between epithelial cells and mesenchymal cells. The parotid gland is the most common site $(90 \%)$. Approximately, $8 \%$ of PA involves the minor salivary glands. As far as intraoral salivary glands are concerned, palate $(42.63 \%)$ is the most affected site followed by lip (10\%), buccal mucosa $(5.5 \%)$, retromolar area $(0.7 \%)$, and lastly affecting the floor of the mouth. Palatal PA presents clinically as a painless, slow-growing mass

\section{Case report}

A 45-year-old male patient came to the Velss Dental care with the complaint of pain, sensitivity, and swelling in the right upper first molar region. History reveals that the patient had severe attrition in the right upper first molar with swelling in the palate which gradually increased in size. As there was no difference in swelling after the extraction of the upper right first molar, the patient reported back. His intraoral examination revealed a painless swelling in the palate (right side) which was oval-shaped,

CT scan and MRI revealed a $16 \mathrm{~mm} \times 14 \mathrm{~mm}$ spherical lesion with a small bone defect in the medial aspect of the alveolus at the level of the first molar region adjacent to the lesion with no evidence of erosion of hard palate or found on the junction of the hard and soft palate which extends in a posterolateral direction that does not cause ulceration of the overlying mucosa. But these tumors are known to cause underlying bone erosion. Pleomorphic adenomas may occur at any age, but mainly they affect patients in the fourth, fifth, and sixth decades. Forty percent of them are male, sixty percent are female. The potential risk of the PA becoming malignant is about $6 \%$

circumscribed, measuring about $1.5 \mathrm{~cm}$ in diameter extending from the mesial aspect of canine to mesial aspect of the first permanent molar, 4-5mm below the marginal gingiva in the palate till the mid palatine raphe. On Palpation it was smooth, firm in consistency, and fixed to underlying mucosa. The overlying mucosa was not ulcerated but stretched and appeared to be shinier than other aspects of the palate.

extension into the maxillary sinus or nasal cavity. The nasal septum deviated to the left.

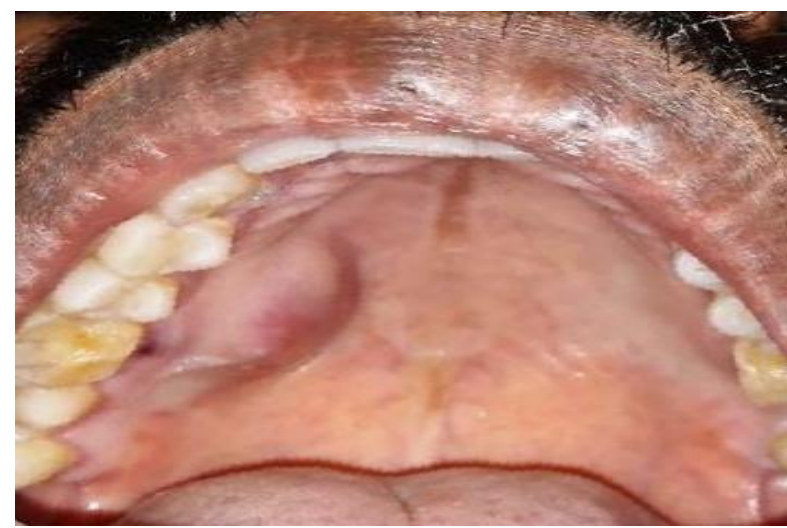

Figure1: Preoperative intra oral view showing swelling in the right side of palate 


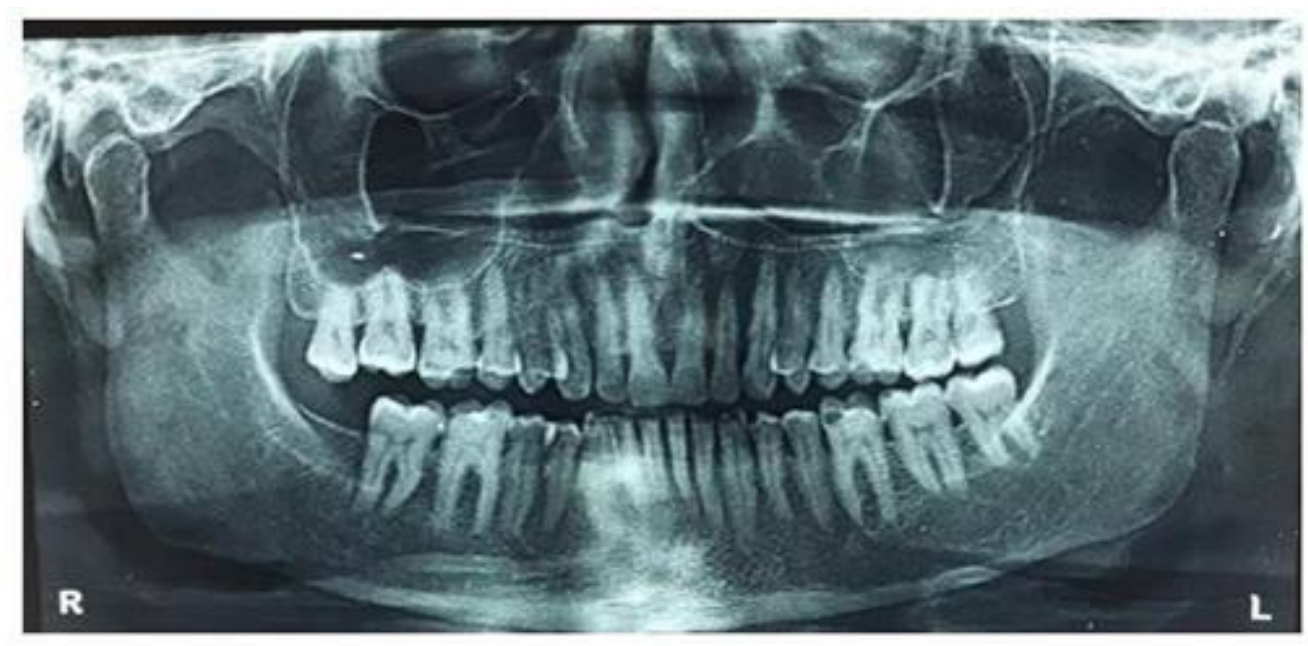

Figure 2: OPG Revealing radiopaque lesion in relation to 16, 17 The differential diagnosis for this case includes palatal abscesses, odontogenic and nonodontogenic cysts, soft tissue tumors such as fibroma, lipoma, and lymphoma as well as other salivary gland tumors. Histology suggested benign salivary gland neoplasm.

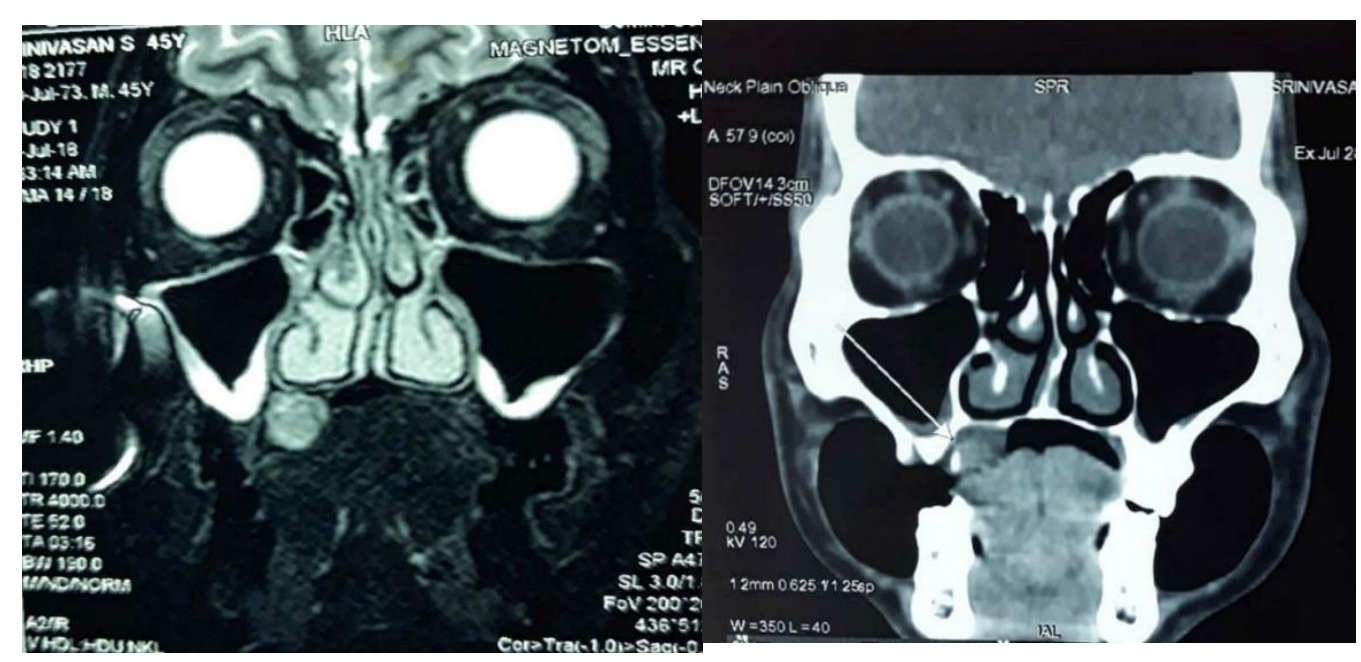

Figure 3: (a \& b). MRI showing no evidence of erosion of hard palate and extension into maxillary sinus or nasal cavity

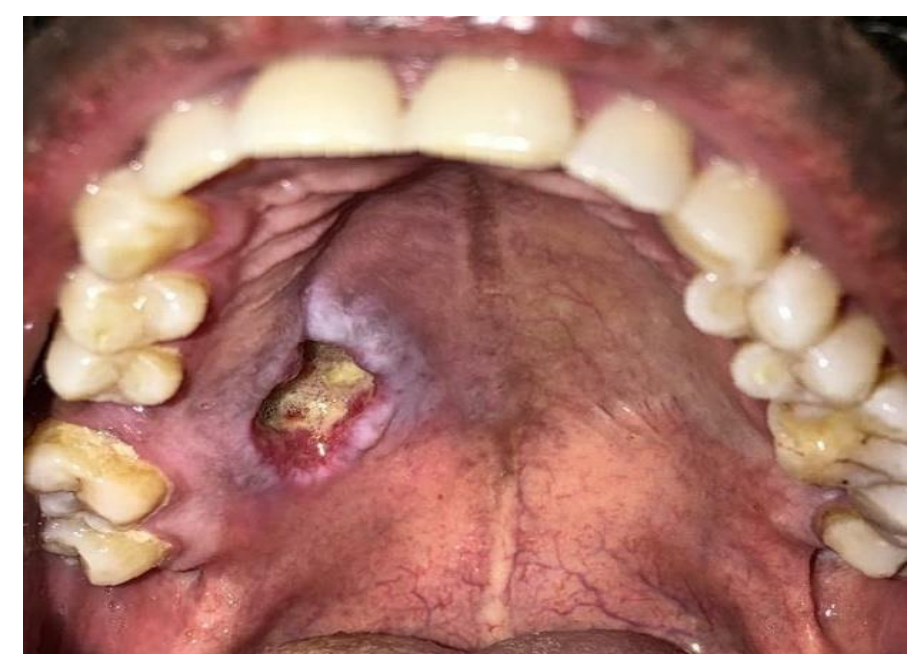

Figure 4: Postoperative intraoral view showing excision of the swelling

The lesion was treated by surgical excision and the excised tissue was sent for histopathological examination which further confirmed the diagnosis.

\section{Discussion}

The diagnosis of PA is established based on history, physical examination, and histopathology. CT scan and magnetic resonance imaging can provide information on the location and size of the tumor and extension to surrounding superficial and deep structures. The treatment is strictly wide local excision with the removal of periosteum or bone if they are involved.

\section{Conclusion}

Since the majority of minor salivary gland tumors are reported to be malignant, careful history, patient evaluation, histopathological and radio imaging are advised. With adequate surgical excision, the tumor usually
Minor salivary gland tumors are detected and treated earlier than major salivary gland tumors because of difficulty in mastication, speech, and swallowing. If the overlying mucosa is ulcerated malignancy is suspected which is not seen in the present case.

does not recur. Hence, most recurrences can be attributed to inadequate surgical techniques. A long-term follow-up is warranted because of the recurrence even after several years of initial excision. 